consulted for Kite/Gilead, MSD, Novartis, and Takeda. J.P. reports personal fees from Pfizer, Alexion, Apellis, BMS, Celgene, Novartis, Hexal AG, Boehringer Ingelheim, and Chugai Pharma outside the submitted work. M.S.H. reports financial support of educational meetings at the Carl-Thiem-Klinikum Cottbus, Germany, by Janssen-Cilag GmbH, Takeda Pharma Vertrieb GmbH \& Co. KG, Novartis Pharma Oncology, Pfizer Pharma GmbH, Roche Pharma AG, Vifor Pharma Deutschland GmbH, and Celgene GmbH. M.J.G.T.V. has served at the speakers' bureau of Akademie für Infektionsmedizin, Ärztekammer Nordrhein, Astellas Pharma, Basilea, Gilead Sciences, Merck/MSD, Organobalance, and Pfizer; has received research funding from 3M, Evoinik, Glycom, Astellas Pharma, DaVolterra, Gilead Sciences, MaaT Pharma, Merck/MSD, Morphochem, Organobalance, and Seres Therapeutics; and is a consultant to Alb-Fils Kliniken GmbH, Arderypharm, Astellas Pharma, Ferring, DaVolterra, MaaT Pharma, and Merck/MSD. L.M.B. reports lecture honoraria from Astellas and MSD and travel grants from 3M and Gilead. E.S. reports no conflicts of interest relevant to this article.

\section{References}

1. Magill SS, O'Leary E, Janelle SJ, et al. Changes in prevalence of healthcareassociated infections in US hospitals. N Engl J Med 2018;379:1732-1744.

2. Battaglia CC, Hale K. Hospital-acquired infections in critically ill patients with cancer. J Intensive Care Med 2019;34:523-536.

3. Hallam C, Jackson T, Rajgopal A, Russell B. Establishing catheter-related bloodstream infection surveillance to drive improvement. J Infect Prev 2018;19:160-166.

4. Surveillance of nosocomial infections as well as the detection of pathogens with special resistance and multi-resistance [in German]. Bundesgesundheitsblatt Gesundheitsforschung Gesundheitsschutz 2013;56:580-583.
5. Parienti JJ, Mongardon N, Mégarbane B, et al. Intravascular complications of central venous catheterization by insertion site. N Engl J Med 2015; 373:1220-1229.

6. Biehl LM, Huth A, Panse J, et al. A randomized trial on chlorhexidine dressings for the prevention of catheter-related bloodstream infections in neutropenic patients. Ann Oncol 2016;27:1916-1922.

7. Hentrich M, Schalk E, Schmidt-Hieber M, et al. Central venous catheterrelated infections in hematology and oncology: 2012 updated guidelines on diagnosis, management and prevention by the Infectious Diseases Working Party of the German Society of Hematology and Medical Oncology. Ann Oncol 2014;25:936-947.

8. Frieden TR. Evidence for health decision making-beyond randomized, controlled trials. N Engl J Med 2017;377:465-475.

9. Schalk E, Hanus L, Färber J, Fischer T, Heidel FH. Prediction of central venous catheter-related bloodstream infections (CRBSIs) in patients with haematologic malignancies using a modified Infection Probability Score (mIPS). Ann Hematol 2015;94:1451-1456.

10. Schalk E, Toelle D, Schulz S, et al. Identifying haematological cancer patients with high risk for central venous catheter (CVC)-related bloodstream infections at the time point of CVC insertion (abstract P2556). Presented at the $29^{\text {th }}$ European Congress of Clinical Microbiology and Infectious Diseases, April 13-16, 2019, Amsterdam, The Netherlands.

11. Wolf HH, Leithauser M, Maschmeyer G, et al. Central venous catheterrelated infections in hematology and oncology: guidelines of the Infectious Diseases Working Party (AGIHO) of the German Society of Hematology and Oncology (DGHO). Ann Hematol 2008;87:863-876.

\title{
A pseudo-outbreak of Legionnaires' disease in an acute-care hospital
}

\author{
Roland Schulze-Röbbecke (1) \\ Department of Infection Control and Infectious Diseases, RWTH University Hospital Aachen, Aachen, Germany
}

To the Editor-Epidemics of Legionnaires' disease (legionellosis) may involve large numbers of cases, with case-fatality rates of about $10 \%$ overall, and $25 \%$ in healthcare-associated cases. ${ }^{1}$ The sources of legionellosis outbreaks are usually building water systems and devices, including potable water and cooling towers; therefore, any suspicion of a legionellosis outbreak must prompt efforts to identify the source and to stop further transmission.

This is a report on a suspected healthcare-associated legionellosis outbreak in an acute-care hospital involving 10 inpatients who tested positive for urinary antigen of Legionella pneumophila serogroup 1 between January and April 2017. Of 160 urinary antigen tests performed during this time period, 10 yielded positive results (positivity rate, 6.3\%). In the previous year, only 1 of 76 tests had yielded a positive result (positivity rate, 1.3\%).

The public health authority was notified and extensive testing of water samples for Legionellae was ordered. These tests only yielded L. pneumophila of a serogroup other than 1. Patient data showed that 5 patients had signs of pneumonia on admission:

Author for correspondence: Roland Schulze-Röbbecke, PhD, Department of Infection Control and Infectious Diseases, RWTH University Hospital Aachen, Pauwelsstraße 30, 52074 Aachen, Germany. Email: roland.schulze-roebbecke@rwth-aachen.de

Cite this article: Schulze-Röbbecke R. (2020). A pseudo-outbreak of Legionnaires' disease in an acute-care hospital. Infection Control \& Hospital Epidemiology, 41: 256-257, https://doi.org/10.1017/ice.2019.365
1 patient had nonrespiratory signs consistent with legionellosis on admission, and 4 patients did not present any signs of pneumonia during their hospital stay. At the time of intervention, all but 1 patient had been discharged. A urine sample of the last patient was divided into 2 portions. One portion was sent to laboratory A, which had issued the positive test results, and the other portion was sent to laboratory $\mathrm{B}$. In laboratory $\mathrm{A}$, the urine sample again tested positive, whereas in laboratory $\mathrm{B}$, the sample tested negative. Confronted with these findings, laboratory A reported having switched to a new urinary antigen test early in 2017, which later turned out to be of poor specificity. After a healthcareassociated outbreak had been ruled out, public health officials ruled out an outbreak altogether, either because legionellosis was not confirmed in the patients presenting signs of pneumonia or because no epidemiological link was found.

In conclusion, the presumptive healthcare-associated legionellosis outbreak caused considerable unrest within the hospital and among the public health authorities, but it proved to be a pseudo-outbreak. Pseudo-outbreaks (or pseudo-epidemics) are real clusters of false infections or artifactual clusters of real infections. ${ }^{2}$ The pseudo-outbreak described here was caused by false-positive urinary-antigen test results. Community pseudooutbreaks of this kind have been reported previously. ${ }^{3,4}$ In our case, the pseudo-outbreak was complicated by assuming healthcare 
association. It could have been avoided if some well-established rules had been observed: (1) Every cluster of positive tests for an infectious disease must cause laboratory personnel and clinicians to communicate and confirm true infections, for example, by considering individual symptoms and signs and by using a second diagnostic test. (2) Before establishing an outbreak, a pseudooutbreak must always be excluded. And (3) diagnosis of an infectious disease some time after hospital admission does not imply that it was acquired in the hospital. The incubation period does not precede the time of diagnosis but the time of onset of the disease.

Acknowledgments.

Financial support. No financial support was provided relevant to this article.
Conflicts of interest. The author reports no conflicts of interest relevant to this article.

\section{References}

1. Fact sheet: what clinicians need to know about Legionnaires' disease. Centers for Disease Control and Prevention website. https://www.cdc.gov/legionella/ downloads/fs-legionella-clinicians.pdf. Accessed December 9, 2019.

2. Weinstein RA, Stamm WE. Pseudo-epidemics in hospital. Lancet 1977;2: 862-864.

3. Regan CM, Syed Q, Mutton K, et al. A pseudo-community outbreak of Legionnaires' disease on Merseyside: implications for investigation of suspected clusters. J Epidemiol Commun Health 2000;54:766-9.

4. Rota MC, Fontana S, Montaño-Remacha C, et al. Legionnaires' disease pseudo-epidemic due to falsely positive urine antigen test results. J Clin Microbiol 2014;52:2279-2280. 